\title{
L'EXPÉRIENCE SPECTATORIELLE COMME TECHNIQUE DE SOI RACIALISANTE
}

\author{
Maxime Cervulle $^{1}$
}

Depuis le début des années 2000, l'émergence soutenue de la thématique de la diversité au sein des sphères publiques française et européenne a notamment renouvelé le débat scientifique et politique autour des discriminations ethnoraciales. Le taux de représentation des dites « minorités visibles », dans les secteurs audiovisuels ou cinématographiques français, a en particulier conduit à une multiplication des rapports et dispositifs publics compétents. Cette exigence de représentativité et d'inclusion désormais adressée au cinéma et à l'audiovisuel français pose la question des conséquences sociales du déficit de représentation des minorités ethnoraciales, notamment s'agissant de la reproduction des rapports sociaux de race asymétriques. À partir et d'une certaine manière à rebours du débat français sur la diversité, cette recherche vise à interroger les termes des modes de subjectivation ethnoraciale des publics. Il s'agit ainsi de penser les façons par lesquelles l'expérience spectatorielle peut constituer le support d'une construction de soi, ainsi que de s'interroger sur l'incidence de la " pâleur des écrans » sur le rapport à soi ainsi constitué. En se penchant sur les types d'identification par lesquelles les publics éprouvent le cinéma, il s'agit de penser le rôle que peut jouer cette expérience dans la formation

1 Université Paris 1 Panthéon-Sorbonne, France.

Recherches en communication, $\mathrm{n}^{\circ} 36$ (2011). 
et stylisation des identités ethnoraciales. À partir d'une étude de réception inspirée du champ anglo-saxon des Critical White Studies et constituée d'entretiens individuels et collectifs auprès de publics socialement identifiés en tant que « blancs » sont ainsi décrites les façons par lesquelles le cinéma peut opérer en tant que technique de soi racialisante.

Depuis le début des années 2000, l'émergence soutenue de la thématique de la diversité au sein des sphères publiques française et européenne a notamment renouvelé le débat scientifique et politique autour des discriminations ethnoraciales. Le taux de représentation des dites " minorités visibles », dans les secteurs audiovisuels ou cinématographiques français, a en particulier conduit à une multiplication des rapports et dispositifs publics compétents. Cette exigence de représentativité et d'inclusion désormais adressée au cinéma et à l'audiovisuel français pose la question des conséquences sociales du déficit de représentation des minorités ethnoraciales, notamment s'agissant de la reproduction des rapports sociaux de race asymétriques. À partir et d'une certaine manière à rebours du débat français sur la diversité, cette recherche vise à interroger les termes des modes de subjectivation ethnoraciale des publics. Il s'agit ainsi de penser les façons par lesquelles l'expérience spectatorielle peut constituer le support d'une construction de soi, ainsi que de s'interroger sur l'incidence de la « pâleur des écrans $~^{1}$ sur le rapport à soi ainsi constitué.

\section{L'expérience spectatorielle au prisme des rapports sociaux de race}

Si la notion de " race » désigne un rapport social notamment caractérisé par la formation d'identités ou d'assignations identitaires sur lesquelles se fondent rapports de pouvoir et discriminations, il apparaît nécessaire d'en penser les implications aussi bien pour les groupes minorés que pour ceux en situation hégémonique. C'est à ce projet

1 Pour reprendre les termes de l'avis du Haut-conseil à l'intégration (Hci), rendu au Premier ministre en 2005, et du colloque «Écrans pâles » qui l'a précédé, co-organisé par le Hci, le Conseil supérieur de l'audiovisuel (Csa) et le Fonds d'action de soutien pour l'intégration et la lutte contre les discriminations (Fasild, aujourd'hui Acsé), qui s'est tenu le 26 avril 2004 à l'Institut du Monde arabe à Paris. 
que ce sont attelés les Critical White Studies en étudiant la formation sociale des identités blanches et leur articulation avec le racisme systémique (Cervulle, 2011a). En nous inspirant de ce champ, nous prenons pour objet non pas les minorités ethnoraciales, mais les publics en situation hégémonique vis-à-vis des rapports sociaux de race et dont la représentation sur les écrans serait prédominante. Il s'agit ainsi de se demander si la blancheur des écrans, notamment postulée par les pouvoirs publics ${ }^{1}$, contribue à la reproduction d'identités, de pratiques ou de conduites participant du racisme systémique?

Notre recherche se concentre sur les processus de subjectivation et d'identification raciales telles que vécues par des spectateurs de cinéma identifiés comme « blancs $»^{2}$. Ceci requiert à l'évidence d'importantes précautions afin notamment d'éviter le piège de la réification de la catégorie « blanc ». La prévention de ce risque cognitif aussi bien que politique passe ici par deux positions, l'une théorique, l'autre méthodologique. La première consiste en un constructivisme social, selon lequel les identités blanches ne renverraient en aucune façon à un certain type d'origine, mais plutôt à des formes d'auto-identifications et d'allo-identifications par lesquelles des acteurs sociaux bénéficient de gratifications sociales au sein d'une société structurellement marquée par la discrimination ethnoraciale. Le concept de «blanchité » ne désigne pas tant une propriété de certains corps qu'une expérience sociale caractérisée par une situation hégémonique au sein des rapports de race (Ahmed, 2004). Il n'est donc pas question de décrire a priori des enquêtés en tant que blancs. Il s'agit plutôt d'appréhender dans un cadre compréhensif la manière dont ces derniers vivent leur situation au sein des rapports sociaux de race. La catégorie «blanc » est donc mise à l'épreuve du terrain et de l'expérience qu'en font les enquêtés

1 Si plusieurs rapports du Csa et le « baromètre de la diversité » co-piloté par le Csa et l'Institut français d'opinion publique (Ifop) permettent d'établir précisément la part des «blancs » et des «non-blancs » sur le petit écran, il n'en est pas de même pour le secteur cinématographique, assez peu doté en ce domaine. Le postulat d'une sous-représentation d'une partie de la population au sein du cinéma français, principalement présenté dans les documents de communication et rapports de la commission « Images de la diversité » du Centre national de la cinématographie et de l'image animée $(\mathrm{Cnc})$, doit donc avant tout être considéré comme une hypothèse de travail. Voir Cervulle, 2011b.

2 Nous choisissons d'écrire les termes «blanc» et « noir » en minuscule, contrairement à l'usage français. Ce choix typographique vise à éviter l'effet potentiellement réifiant de la « majesté » de la majuscule. 
que nous avons rencontrés à l'occasion de 16 entretiens semi-directifs individuels et de 2 entretiens collectifs (ou focus group).

Les 22 enquêtés de cette recherche ont été recrutés par la méthode dite " de proche en proche ", soit par effet de recommandation. L'ensemble des enquêtés réside à Paris intra-muros. Leur âge moyen est de 31 ans, avec un âge maximal de 45 ans et minimal de 22 ans. On dénombre parmi la population d'enquête 11 femmes et 11 hommes. Les enquêtés se répartissent dans cinq des huit principales catégories socioprofessionnelles telles que définies par la nomenclature de niveau 1 du PCS 2003 de l'INSEE ${ }^{1}$ : quatre sont sans activité professionnelle et terminent leur études ; quatre sont employés ; deux sont artisans ou commerçants et chefs d'entreprise ; cinq sont cadres ; sept relèvent de la catégorie des professions intermédiaires. La représentativité de la population d'enquête achoppe concernant les inactifs, retraités et chômeurs qui à l'échelle de la population de Paris totalisent, selon les données établies lors du recensement de 2007, 19,7\% de la population âgée de 15 à 64 ans. On peut considérer cette population d'enquête comme relativement significative en ce qui concerne les actifs parisiens âgés de 22 à 45 ans. Rapportée aux données établies par la statistique publique lors du recensement de 2007, on perçoit que la répartition des actifs par PCS dans l'enquête ne donne pas une image trop faussée de la ventilation réelle par professions et catégories socioprofessionnelles à Paris² Seuls les ouvriers (10,2\% de la population active à Paris en 2007) et agriculteurs et exploitants ( $0 \%$ de la population active à Paris) ne sont pas représentés dans notre panel d'enquêtés. Si l'échantillon n'est pas pleinement significatif, il reste néanmoins relativement pertinent dans sa ventilation par PCS. On peut d'ailleurs expliquer l'absence de représentation ouvrière dans la population d'enquête par deux facteurs, l'un externe, l'autre interne à l'enquête. Il faut d'abord souligner la faible présence des ouvriers dans le département de Paris (10,2\% des actifs) en comparaison à leur représentation nationale $(22,9 \%$ de la population active en France). Ensuite, cette absence de la population d'enquête

$1 \mathrm{http}: / /$ www.insee.fr/fr/methodes/default.asp?page=nomenclatures/pcs2003/liste n1.htm (consulté le 25 septembre 2010).

$211 \%$ d'artisans, commerçants et chefs d'entreprise dans notre population d'enquête pour $4,7 \%$ dans le département de Paris ; $28 \%$ de cadres et professions intellectuelles supérieures dans notre enquête pour $31,9 \%$ dans le département ; $39 \%$ de professions intermédiaires pour $25,6 \%$ dans le département ; $22 \%$ d'employés dans l'enquête pour $27,6 \%$ parmi les actifs d'Île-de-France. 
provient-t-elle sans doute aussi d'un biais de la méthode de recrutement « de proche en proche » qui tend à reconduire des effets de ségrégation sociale. Nous pouvons néanmoins contraster ce point en affirmant que l'appartenance à une catégorie socio-professionnelle n'a eu qu'une incidence faible, si ce n'est nulle, relativement à l'appréhension de l'expérience spectatorielle ou de l'expérience ethnoraciale dans le cadre de cette enquête.

L'enquête de terrain menée dans le cadre de cette recherche vise à identifier les conditions d'émergence de cadres interprétatifs racialisés tout autant qu'à étudier la formation de l'identité ethnoraciale des publics. La situation d'entretien que nous avons élaborée visait à rendre saillant pour les enquêtés l'opérateur interprétatif « race » afin d'étudier en contexte les modalités d'énonciation et de production du sens des publics vis-à-vis de leur trajectoire de spectateur et de leur possible identité ethnoraciale. C'est pourquoi nous avons choisi de structurer le guide d'entretien selon trois phases successives. La première phase consiste à questionner l'enquêté sur sa « carrière » de spectateur (premier film marquant, films préférés, personnages auxquels il s'est identifié, etc.). La deuxième phase porte d'abord, de façon assez libre, sur la définition identitaire du spectateur; puis consiste à lui suggérer une possible identification ethnoraciale ( " vous considérez-vous comme blanc ?») et à lui proposer une conception du racisme comme systémique. La deuxième phase introduit une rupture dans l'entretien en induisant la possibilité d'une interprétation contextuelle de l'enquêté vis-à-vis de sa trajectoire et de ses expériences de spectateur en tant que situées racialement. Pour finir, la troisième phase revient sur ses expériences de spectateur et sur le rôle joué par son identité dans les modes de lecture qu'il met en œuvre.

Cette stratégie d'enquête a été conçue pour répondre à la difficulté méthodologique majeure qu'oppose une recherche sur la blanchité : non pas l'inanité de cette catégorie pour les enquêtés (qui face à la question explicite de leur identification possible en tant que «blanc » répondent très majoritairement par l'affirmative), mais le silence convenu qui l'entoure habituellement ${ }^{1}$. Sa conception repose d'autre part sur une compréhension pragmatique de l'entretien en tant que situation de coproduction discursive permettant l'émergence d'une position énonciative - en l'occurrence, ici, d'une position énonciative blanche. C'est

1 Ruth Frankenberg avait déjà relevé cette difficulté dans White Women, Race Matters (1993). 
donc un processus d'énonciation, d'interprétation et d'expression identitaire que fait émerger l'entretien. En introduisant la possibilité d'un mode de lecture non pas racialiste mais prenant en considération la position de l'enquêté dans les rapports sociaux de race, le guide d'entretien établit des conditions rendant possible la mobilisation des normes et conventions propres à une communauté interprétative racialisée. Ainsi, si l'enquête se penche sur la manière dont les identités blanches des publics se font en rapport à leurs expériences filmiques, elle joue elle-même, sur le moment, un rôle dans la formation de cette identité et de la communauté interprétative imaginaire qui lui est liée. Cela ne revient en aucune façon à dire que l'entretien produit de toutes pièces une catégorie socialement et cognitivement inexistante. L'entretien tend plutôt à jouer le rôle d'élément déclencheur, permettant de recréer en situation " de laboratoire » le processus de cristallisation de l'énonciation et l'interprétation racialisées telle qu'elles peuvent s'exprimer diversement dans des cas concrets.

L'hypothèse selon laquelle l'hégémonie blanche au sein de la représentation cinématographique participerait de la reproduction du racialisme et du racisme se fonde sur une vision constructiviste du cinéma. "Le cinéma n'est pas seulement réaliste parce qu'il capte la réalité, écrit Dominique Chateau, pas simplement parce qu'il en fait partie, mais surtout parce qu'il contribue à sa configuration et façonne son devenir. » (Chateau, 2006, p. 55) Penser la configuration du réel par le cinéma peut notamment impliquer de considérer les manières par lesquelles il participe de la reproduction de rapports sociaux de race asymétriques. Un tel cadre de recherche pourrait nous porter à étudier l'idéologie raciale de textes ou discours filmiques ${ }^{1}$ mais nous choisissons plutôt de nous situer du côté de la réception, afin de faire apparaître certains des modes d'articulation des expériences spectatorielle et ethnoraciale. Une analyse systématique des données recueillies grâce aux entretiens nous a permis de faire émerger trois idéaux-types de la relation entre ces deux pôles de l'expérience tels que vécus et appréhendés par les spectateurs. Nous nous concentrerons ici sur l'un de ces types, permettant d'éclairer singulièrement la subjectivation ethnoraciale par l'expérience spectatorielle. L'analyse typologique, qui relève d'un projet de sociologie compréhensive, doit être saisie comme permettant d'élaborer des descripteurs conceptuels, des catégories

1 De nombreuses recherches existent sur cette question, dont l'ouvrage fondateur de Richard Dyer (1997) ou celui de Linda Williams (2001). 
heuristiques (Schnapper, 2005). En ce sens, 1'idéal-type présenté doit permettre de cartographier les systèmes de croyances usuels des acteurs sociaux. Ces croyances, au sens de William James (2007), sont au cœur du processus de formation du sujet blanc, et donc d'une certaine expérience de soi marquée par le poids des rapports sociaux de race.

La méthode d'analyse typologique se révèle particulièrement pertinente dans le cas d'une étude visant à caractériser la relation entre plusieurs phénomènes sociaux (ici l'articulation entre expérience spectatorielle et expérience ethnoraciale). Elle permet en effet de dégager diverses modalités de cette relation, faisant émerger autant d'idéauxtypes, c'est-à-dire de modèles logiques schématisant les caractéristiques générales de la relation donnée. Comme le souligne Weber, les idéaux-types ne renvoient pourtant en aucune façon ni à des catégories normatives idéales, ni à des positions occupées telles quelles par les acteurs sociaux. Ils désignent plutôt des catégories idéelles permettant de saisir ce que Weber qualifie comme les « relations conceptuelles des problèmes. $\gg{ }^{1}$ Ils permettent de dresser une cartographie conceptuelle éclairant la réalité empirique sans la refléter mécaniquement et parfaitement.

\section{Technologies de race}

Nous présentons ici un portrait critique du « spectateur apprenant » : un idéal-type qui permet de saisir le potentiel pédagogique de l'expérience spectatorielle, au sens où celle-ci peut constituer le socle d'une sensibilisation à l'asymétrie des rapports sociaux de race, aussi bien qu'en ce qu'elle peut tendre à un apprentissage culturel de ce que signifie être blanc au sein d'une société racialisée. La définition du cinéma portée par les publics et sur laquelle elle repose met donc l'accent sur ses fonctions pédagogiques et de socialisation. Elle est caractérisée par une conception de l'expérience spectatorielle selon laquelle celle-ci permettrait d'apprendre sur soi ou le monde alentour, y compris à propos des rapports sociaux de race.

Ce type de relation du spectateur au film recouvre deux modes différents d'identification filmique, que nous pouvons appeler « identification présentielle narrative » et « identification prospective esthé-

1 Max Weber, Essai sur la théorie de la science, Paris, Plon, 1992, p. 142, cité dans D. Schnapper, «L'analyse typologique », ibid., p. 299. 
tique ». L'identification présentielle conjugue diverses formes de reconnaissance de soi dans l'espace filmique et, ainsi, de validations volontaires de caractéristiques sociales ou idiosyncrasiques. L'identification présentielle et sa mobilisation discursive après-coup, notamment en situation d'entretien, viennent sanctionner un vécu, une caractéristique identitaire, une appartenance. Loin de figer une identité, le film avec lequel ce type de proximité prend place est présenté comme une occasion d'apprentissage sur soi et son univers social immédiat. L'identification présentielle narrative s'attache au récit et aux personnages qui l'animent. Elle se situe du côté d'une « fictionnalisation » de soi permettant de réévaluer des évènements vécus ou situations concrètes à l'aune de leur mise en récit.

L'identification prospective est parfaitement résumée par les propos d'un de nos enquêtés : "c'est une projection : ce que j'aimerais faire, ce à quoi j'aspire ». Il s'agit en un sens d'une stylisation de soi imaginaire soutenue par l'entrée dans un univers fictionnel. L'identification prospective et son expression discursive tendent à marquer les souhaits et aspirations du spectateur, tant en termes de qualités personnelles que d'expériences de vie. Ce type d'identification autorise ainsi une véritable stylisation d'un soi en construction. Là où l'identification présentielle liait le spectateur au film dans un présent et un passé communs, l'identification prospective oriente ainsi le spectateur vers des horizons futurs souhaités et représentés à l'écran. Là où la première orientait l'attention du spectateur vers la dimension narrative, il porte ici son regard vers le projet esthétique du film.

Le « spectateur apprenant » peut mobiliser alternativement ces deux modes d'identification. Lorsqu'il vit une expérience spectatorielle sur le registre de l'identification présentielle narrative, la reconnaissance de soi au sein de la diégèse peut prendre la forme d'une identification explicite en tant que «blanc ». De façon intéressante, cette identification s'exprime le plus souvent au travers d'un sentiment de culpabilité vis-à-vis de gratifications qu'il juge immérités et entièrement dues à sa position au sein des rapports sociaux de race. Concernant ce sentiment, l'un des enquêtés déclare : " J'ai accès à certaines choses sans faire réellement d'effort [...], il y a toujours la culpabilité d'être privilégié. » Avec la culpabilité, le potentiel sentiment de responsabilité - éthique ou politique - est traduit dans un registre émotionnel individuel qui s'éloigne d'autant du terrain où il devrait s'exprimer qu'il recentre la focale sur la singularité d'un sujet blanc spécifique. Des expériences en psychologie sociale, conduites aux États-Unis par Aarti Iyer et. 
al. (2003), ont démontré que le sentiment de culpabilité, qui indique une certaine reconnaissance des inégalités, peut conduire les blancs à soutenir les politiques de dédommagement ou de compensation, mais n'entraîne pas pour autant leur adhésion en ce qui concerne les actions publiques visant l'égalité des chances et la création d'opportunités nouvelles pour les minorités ethnoraciales (notamment les programmes d'affirmative action ou, selon l'imparfaite traduction française, de « discrimination positive »). Le sentiment de culpabilité apparaît ainsi dans ce contexte comme visant non pas tant à corriger une situation sociale jugée injuste selon quelque position éthique normative, qu'à restaurer une certaine image de soi. Cela est apparu avec force dans les entretiens, certains enquêtés soulignant la honte ou la colère qu'ils ont pu ressentir dans des situations où il leur a semblé bénéficier d'avantages dus à leur couleur de peau. Cette honte ou cette colère ne sont pas directement dirigées à l'encontre des discriminations ethnoraciales qui les ont avantagés, mais contre le fait qu'ils n'aient pas été choisis pour les bonnes raisons, c'est-à-dire selon les critères méritocratiques usuels et socialement acceptables dans le cadre républicain. Comme le dit une enquêtée : " c'est énervant [...] d'accéder à certaines choses pour des faits qu'on ne peut pas choisir, genre sa couleur de peau ». Le recentrement émotionnel sur soi que suppose le sentiment de culpabilité tend à engendrer des réponses individuelles isolés, plutôt que politiques ou collectives.

Au-delà d'une simple reconnaissance de soi, l'identification présentielle narrative qu'éprouvent les spectateurs face à un film peut également renvoyer à une forme de « fictionnalisation » de soi permettant de prolonger dans la fiction une situation sociale vécue. Elle ouvre parfois la voie à des envies de changement, principalement à un niveau personnel et individuel. L'identification à un personnage ou à une situation narrative peut permettre, par exemple, de reconsidérer une situation, comme dans le cas d'une enquêtée dont les identifications filmiques aux récits de conflits familiaux l'ont poussée à relativiser la gravité de ses propres difficultés familiales. Ce type d'identification peut également susciter des vocations, et donc induire un changement non pas simplement en termes de perception mais d'orientation de l'action. Cela fut par exemple le cas avec une autre enquêtée qui voit rétrospectivement en Indiana Jones une inspiration pour son goût de la science en général et sa carrière en géologie en particulier. En ce qui concerne les rapports sociaux de race, le changement d'orientation de l'action que peut inaugurer l'identification présentielle narrative se situe dans le cadre d'une 
action bénéficiant à celui qui l'accomplit. En effet, il s'agit là encore d'une action individuelle qui correspond donc au même type de recentrement sur soi que porte le sentiment de culpabilité.

La relation pédagogique du spectateur au film peut aussi revêtir un autre aspect : celui d'apprendre ce qu'être blanc veut dire au sein d'une société marquée par une racialisation du champ social. Pour Gwendolyn Audrey Foster (2003), le cinéma produirait de façon performative des registres d'identification raciaux, notamment par la répétition itérative de performances de la blanchité - ce qu'elle nomme le "whiteface ». Comme dans la théorie de la «performativité du genre » de Judith Butler (2005), la blanchité constituerait une copie sans original, mais dont la répétition permanente contribuerait à établir l'idée racialiste d'un fondement « naturel» des identités blanches. Les performances blanches s'inscriraient dans un espace cinématographique historiquement marqué par l'exclusion des corps non-blancs : une exclusion qui fut notamment le fait de choix industriels dans l'histoire des techniques photographiques, établissant le visage blanc en étalon de l'esthétique de la lumière (Dyer, 1997, pp. 82-144). Foster souligne également combien le Code de production hollywoodien de 1930 a pu participer de cette exclusion en confondant habilement « moralité » et « blanchité » et en parant la « vertu » attendue d'un vernis racial.

Inscrire la performance blanche dans un processus itératif revient à dire que chacune de ses manifestations enregistre un écart qui la rend ainsi susceptible de changement. Cependant, là où pour Butler les écarts et dissonances relatifs aux performances de genre les rendaient vulnérables à une véritable « politique de la parodie » révélant leur caractère construit, instable et contingent; dans le cas de la blanchité, force est de constater que l'absence de référent stable et unique, et la prolifération de types de "whiteface », participent paradoxalement de sa naturalisation. En effet, comme le souligne Patricia McKee, « dans les termes visuels de la vie publique au vingtième siècle existe un large panel de représentations de la blanchité. Les blancs peuvent donc éprouver leur identité non seulement dans la similarité mais dans la diversité » (1999, p. 14 ; cité dans Foster, 2003, p. 50). Cette expérience intime de la diversité d'identités blanches ouvre la voie à une forme de subjectivation ouverte et négociée, co-produite par les films et leurs publics.

L'un de nos enquêtés relevait qu' " au cinéma, ça semble naturel d'être blanc ». Ses propos signifient combien l'horizon de la représentation est circonscrit par un ensemble d'attentes relatives aux rapports sociaux de race. Un autre, soulignant que certains films ont pu lui 
" apprendre à être blanc ", note quant à lui que s'il avait du mettre « un visage sur Winnie l'ourson, il aurait été blanc ». On voit ainsi à quel point la relégation des minorités ethnoraciales aux marges et hors-champs de l'histoire cinématographique (Bernardi, 2001) participe de la construction du cinéma en tant que site de subjectivation raciale instruisant une sorte de naturalisation de la blanchité. Le sujet blanc devient alors le sujet normatif de la représentation, aussi bien du côté de la production que de celui de la réception. La blanchité est ainsi établie en tant que norme culturelle : le standard de représentation devient catégorie désirable d'identification. Le champ de la représentation cinématographique apparaît ainsi comme étant fondé sur une exclusion constitutive. Hanté par les rapports sociaux de race, qui ont pesé en partie sur l'histoire de son développement technique et industriel, le cinéma produirait ainsi des registres d'identification performatifs qui constituent la blanchité en horizon universel et établissent une définition restrictive de «l'humain » (Dyer, 1997).

Le 《spectateur apprenant » apparaît comme particulièrement sensible aux registres d'identification blanche ouverts par l'interaction entre films et publics. Sa croyance en la capacité socialisante du cinéma, ainsi que les sentiments de culpabilité qu'il éprouve vis-à-vis de son identité ethnoraciale, l'enjoignent à s'investir dans une identité blanche présentée comme positive. Des figures de « héros blanc»-tels que Astérix, Indiana Jones, le personnage de Clint Eastwood dans Gran Torino (Eastwood, 2008) ou celui de Vincent Lindon dans Welcome (Lioret, 2009) - caractérisés par un certain sens moral tout autant que par une attitude paternaliste vis-à-vis des minorités peuvent se situer au cœur d'une relation pédagogique médiée par le film. L'expérience spectatorielle peut ainsi ouvrir un espace de renégociation de l'identité blanche du spectateur, lui permettant de "s'amender » symboliquement, et de se distancer de son sentiment de culpabilité, par un investissement affectif dans des figures héroïques de blancs sauvant à l'écran des minorités ethnoraciales en péril. Le cinéma opèrerait ainsi comme une sorte de " technologie de race », machine de production discursive et représentationnelle par laquelle le spectateur bricole avec les films une identité blanche positive et réformée. Ce concept, qui fait écho à celui de « technologie du genre » de Teresa De Lauretis ${ }^{1}$ (2007, pp.

1 De Lauretis s'appuie elle-même sur le concept de « technologie du sexe » de Michel Foucault, dont elle vise à compléter l'angle mort principal : la non-prise en compte du traitement discursif différencié des hommes et des femmes. 
37-94), permet d'appréhender l'expérience spectatorielle en tant que mode de production du sujet ou, pour le dire avec Foucault (1984), en tant que « technique de soi » racialisante.

L'émergence de multiples registres d'identifications blanches qu'ouvre la collaboration entre films et publics semble reposer sur le sentiment d'une nécessité d'investissement intellectuel, moral et affectif dans une identité blanche positive. Il s'agit en quelque sorte de contester, par une ré-articulation du sens social de la blanchité, les pratiques de violence et de discrimination qu'elle a pu historiquement soutenir. La co-production de registres d'indentifications blanches servirait ainsi un but de mise à distance du sentiment de culpabilité et de responsabilité. L'identification positive aux héros blancs des écrans de cinéma tendrait ainsi à restreindre les conditions d'émergence d'une conscience critique et réflexive vis-à-vis de la violence symbolique et matérielle des rapports sociaux de race.

\section{Dans la peau d'un noir}

Nous venons de nous pencher sur ce que peut produire une expérience spectatorielle guidée par une identification présentielle narrative, mais le « spectateur apprenant» peut aussi exprimer son identification filmique sur un mode prospectif esthétique. Ce mode d'identification qui autorise une forme de stylisation de soi peut s'exprimer dans un désir d'incarnation contradictoire, l'envie de se fondre « dans la peau d'un noir ». Ce fantasme de " blackface $»^{1}$ peut tout d'abord traduire une appréhension des rapports de race qui tend à relativiser leur emprise, à fermer les yeux sur le poids social du racisme systémique. La relativisation de la réalité sociale du racisme est souvent déclarée provenir d'une inexpérience du racisme, inexpérience à laquelle seule « le fait d'être noir » ou d'occuper provisoirement ce point de vue pourrait semble-t-il remédier. On peut notamment sur ce point se référer aux propos d'un des enquêtés déclarant : "Moi je ne suis pas dans la peau d'un noir ou d'un arabe [...], les récits qu'on entend, c'est que c'est vachement dur. Mais j'aurais même du mal à les détailler parce que moi je ne suis pas victime de racisme ». Le cinéma permet de vivre indirectement une

1 Né aux États-Unis au dix-neuvième siècle, le "blackface » consiste en une performance accomplie dans l'espace public par des ménestrels blancs grimés en noirs (William T. Lhamon Jr., 2008). 
telle expérience, de changer littéralement de point de vue, l'identification prospective esthétique permettant au spectateur d'évaluer le caractère vraisemblable des discriminations ethnoraciales. L'expérience spectatorielle vient alors en partie se substituer à l'expérience vécue pour forger une certaine appréhension des rapports de pouvoir au sein de la société. Néanmoins la possibilité que ce type d'expérience par procuration remette en question l'approche relativiste des rapports de race est faible. Elle apparaît en effet minée par une approche récréative du cinéma qui fait de cette expérience d'incarnation d'un point de vue autre une stylisation ludique de soi. Ce recentrement sur le sujet blanc passe par le désir de s'approprier certaines des caractéristiques socialement et culturellement attribuées aux minorités ethnoraciales. L'exemple le plus pertinent est sans doute celui d'un des enquêtés qui exprime, par rapport à son identification aux affects se dégageant de la scène de prêche du personnage de Samuel L. Jackson dans Pulp Fiction (Tarantino, 1994), son « désir de faire peur », « d'avoir la classe » et de « savoir faire de grands discours »-autant de stéréotypes courants pour représenter les africains-américains.

Là où dans le cinéma hollywoodien les minorités ethnoraciales jouent habituellement le rôle d'adjuvants du héros blanc lui permettant de progresser dans le récit et de se révéler à lui-même (Vera \& Gordon, 2003), du côté de la réception, les personnages non-blancs paraissent avoir une fonction similaire. Il semble en effet qu'ils constituent pour les spectateurs blancs, lorsqu'une identification prospective esthétique est opérante, de styliser leur identité par le recours à un certain nombre de stéréotypes ethnoraciaux. Dans The New-Brutality Film, Paul Gormley souligne ainsi combien le cinéma états-unien des années 1990 s'est nourri de discours racialistes relatifs à la prétendue « vitalité » de la culture africaine-américaine, pour sortir le cinéma postmoderne de sa torpeur et élaborer un cinéma " corporel » stylisé, un cinéma du choc sensoriel. Il situe l'œuvre de Quentin Tarantino dans cette veine, soulignant que ses films « visent à produire un choc sensoriel en imitant la réaction immédiate et corporelle que provoquent des images de corps noirs chez des publics blancs. Cette réaction est vécue comme immédiate et corporelle parce qu'elle trouve sa source dans des systèmes de perception visuelle organisés racialement » (Gormley, 2005, p. 13). Du côté des spectateurs, l'identification prospective esthétique à un personnage non-blanc peut reposer sur le même type de mécanisme : se glisser dans la peau d'une minorité ethnoraciale permettrait de ressentir les frissons de l'altérité. Cette expérience spectatorielle met l'accent sur le 
vécu sensoriel et affectif du spectateur et échoue donc à contrer l'appréhension relativiste des rapports de race. Plutôt que de « changer le point de vue » non pas techniquement, mais socialement, elle ne contribue qu'à styliser le soi blanc par le recours à l'exotisme (Cervulle \& ReesRoberts, 2010). En tant que procédé de mise à distance, et donc d'établissement de frontières culturelles, l'exotisme apparaît en lui-même comme un mode de subjectivation et pourrait donc être compris comme l'un des modes d'opération des techniques de soi racialisées.

L'identification aux minorités ethnoraciales pourrait avoir le mérite de proposer une expérience de décentrement - permettant de vivre, pour un instant et par la médiation cinématographique, les rapports sociaux de race de l'autre côté de ce que W. E. B. Du Bois appelle «the veil » (le voile racial). Néanmoins, ce régime d'identification à l'altérité semble tendre à la déresponsabilisation des publics blancs vis-à-vis du racisme systémique dans la mesure où il se manifeste comme une stylisation ludique du sujet blanc. Tout se passe comme si ce sujet blanc reconstruit dans l'expérience de la mobilité identitaire, par l'exercice de l'exotisme, était défini par son aptitude au déplacement, à l'expansion, à l'occupation perpétuelle de nouveaux territoires imaginaires et sociaux. Il est ainsi enclin à une sorte d'universalisation de sa position qui n'est pas sans faire écho à l'universalisme républicain caractérisant le contexte dans lequel il se déploie.

\section{Publics et subjectivation raciale}

Cet « art de la modernité » (Chateau, 2009) qu'est le cinéma n'estil pas le véhicule de cette autre invention de la rationalité moderne qu'est la race ? La maîtrise du récit cinématographique dont fit par exemple preuve le réalisateur états-unien D.W. Griffith, dans un film tel que Naissance d'une nation reconnu désormais comme un véritable manifeste du « suprématisme blanc », et qui constituera la matrice du classicisme hollywoodien (Taylor, 1996), n'était-elle pas aussi maîtrise des signifiants de la race ? Quel rôle joue donc l'expérience spectatorielle dans la formation des identités ethnoraciales? Au vu de l'enquête que nous avons mené, il semble qu'en réception et du point de vue de la construction sociale de la race, le cinéma aurait en premier lieu une fonction subjectivante pour une catégorie de spectateur que nous avons qualifié d' " apprenant ». Si l'assujettissement racial apparaît prendre place en divers domaines et jouer un rôle pour tous les acteurs sociaux, 
dans une société marquée par un racisme systémique, concernant le cinéma l'efficience de ce processus semble requérir certaines dispositions. L'émergence de multiples registres d'identifications blanches qu'ouvre la collaboration entre films et publics reposerait particulièrement sur le sentiment d'une nécessité d'investissement intellectuel, moral et affectif dans une identité blanche positive. Le caractère performatif du cinéma reposerait dans ce cadre sur son aptitude à co-produire avec les publics des registres d'identification valorisants.

Face à la médiation cinématographique des rapports sociaux de race, le spectateur peut être non seulement « apprenant», mais « ignorant » ou « démocrate »; trois termes désignant les trois idéaux-types que l'enquête de terrain nous a permis de construire. Ces types ont en commun de désigner certaines formes de croyances relatives au cinéma et qui déterminent l'expérience spectatorielle. La possibilité d'une subjectivation raciale au cinéma - qui serait notamment liée au déficit de diversité sur les écrans et à la " politique des représentations » (Hall, 2007, pp. 203-214) - repose sur la nature de ces croyances.

Le « spectateur ignorant », pour lequel le cinéma aurait avant tout une fonction divertissante, investit l'expérience spectatorielle comme un lieu de fuite. L'identification filmique n'est pas alors une reconnaissance de soi ou de l'environnement social, mais une forme de distraction, au double sens que le terme comporte. Le spectateur ignorant se distance pour un instant de lui-même, se projette dans une identification prospective esthétique. Cette distraction de soi correspond tout à fait à la conception du monde social de ce spectateur qui ferme les yeux sur les inégalités relatives aux rapports sociaux de race. L'ignorance productive et active de ce spectateur constitue le socle sa subjectivité raciale. Elle donne en effet jour à une identité blanche - que l'on peut définir par les termes «blanchité universalisée »-caractérisée par une occupation du registre de l'universel et la mise en œuvre de stratégies discursives permettant une déresponsabilisation relative vis-à-vis du racisme systémique.

La vision de la société du « spectateur apprenant » est également encline à l'utopie. Elle tend cependant à reconnaître l'existence des discriminations ethnoraciales. Ce spectateur est ainsi caractérisé par un sentiment de culpabilité constitutif de son identité blanche - ce que nous pourrions désigner comme une forme de «blanchité mélancolique ». Le cinéma, qu'il conçoit comme un espace pédagogique de socialisation, peut dans ce cadre et comme nous l'avons souligné, jouer un rôle dans le processus de subjectivation raciale. 
Enfin, le « spectateur démocrate » a de son côté une appréhension du cinéma comme sphère publique, au sens où il reflèterait et ferait émerger les tensions qui parcourent le monde social. Ce spectateur appréhende en effet la société comme terrain conflictuel où s'affrontent idéologies et intérêts concurrents. Son investissement dans l'expérience spectatorielle correspondra ainsi, selon la vision qu'il en a, à un investissement politique, c'est-à-dire attentif aux affaires de la cité. Il portera ainsi attention aux enjeux de représentativité des écrans, notamment mais non exclusivement pour les minorités ethnoraciales. Sa croyance en la capacité du cinéma à servir de support au débat démocratique confine cependant à une certaine idéalisation de la sphère publique comme espace universel. Un idéalisme que vient compléter une vision relativisant l'asymétrie des rapports sociaux de race. L'identité blanche de ce spectateur est fondée sur une croyance en sa puissance d'agir, pensée comme absolue et sans restriction, où la blanchité apparaît sous les traits d'une mobilité et d'une capacité à l'action extensives. Cette identité relève d'une «blanchité morale " par laquelle le sujet blanc se présente en tant que garant moral d'un certain ordre social républicain. Une identité toutefois fondée sur une occultation constitutive : celle des limitations des minorités ethnoraciales dans une société traversée par un racisme systémique.

Considérer, comme nous avons pu le faire, le cinéma comme " technologie de la race » revient à dire qu'il épelle une " grammaire raciale » (Hall, 2007, p. 199) qui nourrit et alimente les différents " répertoires discursifs » (Frankenberg, 1993) par lesquelles s'énoncent et s'éprouvent les identités des publics. L'expérience spectatorielle pourrait ainsi être comprise comme une technique de soi, participant de la formation et stylisation des identités ethnoraciales. Les dispositions des spectateurs « apprenants », enclins à la réarticulation d'une identité blanche déculpabilisée, les rendent plus sensibles à cette grammaire en ce qu'elle permet l'énonciation nouvelle d'une blanchité innocente et héroïque. Les représentations filmiques impactent de façon moindre, et pour ce qui est des rapports sociaux de race, les spectateurs « ignorants » et « démocrates ».

Chacun de ces types de spectateur investit l'expérience spectatorielle comme un espace-temps métaphorisant et soutenant ses croyances vis-à-vis du monde social. Ainsi, le spectateur « ignorant » pour qui le cinéma se résume à un simple divertissement dénué d'enjeux sociaux ou politiques, porte une appréhension utopiste de la société comme terrain non-conflictuel dans lequel il peut se mouvoir librement et sans 
contrainte. Du côté du spectateur « démocrate », le cinéma apparaît comme un reflet de la sphère publique, enregistrant donc les tensions et conflits qui s'y expriment. Enfin, le spectateur « apprenant » est enclin à co-produire avec les films des répertoires discursifs racialistes qui contribueront à la formation et stylisation de son identité ethnoraciale. Le cinéma serait donc pour les publics le support de croyances en partie pré-établies, l'expérience spectatorielle venant souligner et soutenir certaines conduites et certaines perceptions routinières du monde social. Qu'il soit vu comme lieu de manifestation utopique d'une harmonie sociale accomplie, comme reflet d'une société marquée par la conflictualité idéologique, ou enfin comme site pédagogique d'investissement de soi, le cinéma apparaît comme un avatar des modes de conceptualisations de la société (Macé et Maigret, 2005). En ce sens, les sciences de l'information et de la communication comme la sociologie du cinéma et des publics auraient l'avantage de permettre d'exposer et d'objectiver les croyances et représentations ordinaires des acteurs sociaux.

\section{Références}

Ahmed, S. (2004). Declarations of Whiteness : The Non-Performativity of Antiracism. Borderlands e-journal, 3(2). Disponible à : http://www.borderlands.net.au/ vol3no2_2004/ahmed_declarations.html.

Bernardi, D. (Éd.). (2001). Classic Hollywood, Classic Whiteness. Minneapolis : Minnesota University Press.

Butler, J. (2005). Trouble dans le genre. Le féminisme et la subversion de l'identité. Trad. de C. Kraus. Paris : La Découverte, 2006.

Cervulle (2011a). La couleur des épistémologies. Race, politique des savoirs et Critical White Studies. Dans B. Andrieu (Éd.), Le corps du chercheur. Méthodologies immersives. (pp. 163-176). Nancy : Presses universitaires de Nancy.

Cervulle (2011b). La politique de diversité dans les secteurs audiovisuels et cinématographiques. Quels publics pour quelles actions publiques ? Colloque international « Diversité et innovation ». MSH Clermont-Ferrand et Université Blaise Pascal. Disponible à : http://www.lacc.univ-bpclermont.fr/article169.html.

Cervulle, M. \& Rees-Roberts, N. (2010). Homo exoticus. Race, classe et critique queer. Préface de Richard Dyer. Paris : Armand Colin et Ina éditions.

Chateau, D. (2006). Esthétique du cinéma. Paris : Armand Colin.

Chateau, D. (2009). Philosophie d'un art moderne : le cinéma. Paris : L'Harmattan.

De Lauretis, T. (2007). Théorie queer et cultures populaires. De Foucault à Cronenberg. 
Trad. de M.-H. Bourcier. Paris : La Dispute.

Dyer, R. (1997). White. Londres \& New York : Routledge.

Foster, G. A. (2003). Performing Whiteness. Postmodern Re/constructions in the Cinema. New York: State University of New York Press.

Frankenberg R. (1993). White Women, Race Matters : The Social Construction of Whiteness. Minneapolis : Minnesota University Press.

Gormley, P. (2005). The New-Brutality Film. Race and Affect in Contemporary Hollywood Cinema. Bristol \& Portland : Intellect.

Hall, S. (2007). Identités et cultures. Politiques des Cultural Studies. Éd. établie par M. Cervulle. Trad. de C. Jacquet. Paris : Éditions Amsterdam.

Haut-conseil à l'intégration (2005). Diversité culturelle et culture commune dans l'audiovisuel. Avis au Premier Ministre. Disponible à : www.hci.gouv.fr/IMG/doc/ Avis_HCI_audiovisuel.doc.

Iyer, A., Leach, C. W., \& Crosby, F. J. (2003). White Guilt and Racial Compensation : The Benefits and Limits of Self Focus. Personality and Social Psychology Bulletin, 29(1), 117-129.

Lhamon Jr., W. T. (2008). Peaux blanches, masques noires. Performances du blackface de Jim Crow à Michael Jackson. Paris : Cargo.

Maigret, É. \& Macé, É. (Éd.). (2005). Penser les médiacultures. Nouvelles pratiques et nouvelles approches de la représentation du monde. Paris : Armand Colin et Ina éditions.

McKee, P. (1999). Producing American Races : Henry James, William Faulkner, Toni Morrison. Durham : Duke University Press.

Schnapper, D. (2005). La compréhension sociologique. Démarche de l'analyse typologique. Édition revue et augmentée. Paris : Presses universitaires de France.

Taylor, C. (1996). The Re-Birth of the Aesthetic in Cinema. Dans D. Bernardi (Éd.), The Birth of Whiteness : Race and the Emergence of U.S. Cinema. (pp. 15-37). New Brunswick : Rutgers University Press.

Vera, H. \& Gordon, A. (2003). Screen Saviors : Hollywood Fictions of Whiteness. Lanhamn Boulder, New York \& Oxford : Rowman \& Littlefield Publishers Inc.

William, J. (2007). Le pragmatisme. Trad. N. Ferron. Paris : Flammarion.

Williams, L. (2001). Playing the Race Card. Melodramas of Black and White from Uncle Tom to O.J. Simpson. Princeton : Princeton University Press. 